\title{
Relatos de profissionais de saúde no contato com a disciplina de práticas integrativas e espiritualidade em saúde: Vivências, reflexões e ciência
}

\author{
Reports from health professionals in contact with the subject of integrative practices and \\ spirituality in health: Experiences, reflections and science \\ Informes de profesionales de la salud en contacto con el sujeto de prácticas integrativas y \\ espiritualidad en salud: Experiencias, reflexiones y ciencia
}

Recebido: 12/06/2021 | Revisado: 17/06/2021 | Aceito: 21/06/2021 | Publicado: 26/06/2021

Felipe Santos da Silva

ORCID: https://orcid.org/0000-0002-1409-4832

Universidade Estadual de Campinas, Brasil

E-mail: felipe.stos.sva@gmail.com

Natanias Macson da Silva

ORCID: https://orcid.org/0000-0003-1044-7319 Universidade do Estado do Rio Grande do Norte, Brasil

E-mail: nataniassilva@ alu.uern.br

Leonel Francisco de Oliveira Freire

ORCID: https://orcid.org/0000-0002-8734-5292

Universidade do Estado do Rio Grande do Norte, Brasil

E-mail: leonelloliveira93@gmail.com

Jeane Caroline Dantas Ferreira

ORCID: https://orcid.org/0000-0001-8176-9150

Universidade Potiguar, Brasil

E-mail: carolinedantas15@hotmail.com

Maria Aparecida Cursino

ORCID: https://orcid.org/0000-0001-9312-6056

Universidade Estadual de Campinas, Brasil

E-mail: ma.cursino@gmail.com

José Rodrigues Paiva Neto

ORCID: https://orcid.org/0000-0003-2874-2666

Universidade Federal Rural do Semi-Árido, Brasil

E-mail: rodrigues.paiva@ufersa.edu.br

Isabel Cristina Amaral de Souza Rosso Nelson

ORCID: https://orcid.org/0000-0003-4840-6950

Universidade do Estado do Rio Grande do Norte, Brasil

E-mail: isabelrosso@uern.br

\begin{abstract}
Resumo
As Práticas Integrativas e Complementares em Saúde (PICS) têm se constituído um campo fértil para garantir a promoção de saúde e qualidade de vida. Com isso, discussões sobre a espiritualidade têm ganhado relevância social. O presente artigo objetivou tecer reflexões sobre as experiências de profissionais de saúde no transcurso de uma disciplina sobre as PICS integrando-as com a espiritualidade. Tratou-se de um estudo descritivo, de relatos desses profissionais, após a conclusão da disciplina "Tópicos especiais em Pesquisa em Saúde III - Práticas Integrativas e espiritualidade em saúde: Vivências, reflexões e ciência", ofertada pelo Programa de Pós-Graduação em Saúde e Sociedade (PPGSS) da Universidade do Estado do Rio Grande do Norte (UERN). Os resultados evocaram a necessidade de ampliação nas matrizes curriculares nos cursos de graduação em saúde sobre as PICS e espiritualidade. O contato com disciplinas que tematizam as PICS, aspectos da espiritualidade, bem como a relação com uma saúde integral, mostraram-se significativos, conforme o presente estudo objetivou apresentar através da discussão dos relatos.
\end{abstract}

Palavras-chave: Terapias complementares; Promoção da saúde; Espiritualidade; Integralidade em saúde.

\begin{abstract}
Integrative and Complementary Practices in Health (PICS) have constituted a fertile field to ensure the promotion of health and quality of life. As a result, discussions about spirituality have gained social relevance. This article aimed to reflect on the experiences of health professionals in the course of a discipline on PICS, integrating them with spirituality. This was a descriptive study of reports from these professionals, after completing the course "Special Topics in Health Research III - Integrative Practices and Spirituality in Health: Experiences, Reflections and Science",
\end{abstract}


offered by the Programa de Pós-Graduação em Saúde e Sociedade (PPGSS) da Universidade do Estado do Rio Grande do Norte (UERN). The results evoked the need to expand the curriculum in undergraduate health courses on PICS and spirituality. The contact with disciplines that thematize the PICS, aspects of spirituality, as well as the relationship with integral health, proved to be significant, as this study aimed to present through the discussion of reports.

Keywords: Complementary therapies; Health promotion; Spirituality; Integrality in health.

\section{Resumen}

Las Prácticas Integrativas y Complementarias en Salud (PICS) han constituido un campo fértil para asegurar la promoción de la salud y la calidad de vida. Como resultado, las discusiones sobre espiritualidad han ganado relevancia social. Este artículo tuvo como objetivo reflexionar sobre las experiencias de los profesionales de la salud en el curso de una disciplina sobre PICS, integrándolos con la espiritualidad. Se trató de un estudio descriptivo, de los relatos de estos profesionales, luego de la realización de la asignatura "Temas especiales en Investigación en Salud III - Prácticas Integradoras y Espiritualidad en Salud: Experiencias, Reflexiones y Ciencia", impartida por el Programa de Pós-Graduação em Saúde e Sociedade (PPGSS) da Universidade do Estado do Rio Grande do Norte (UERN). Los resultados evocaron la necesidad de ampliar el plan de estudios en los cursos de pregrado en salud sobre PICS y espiritualidad. El contacto con disciplinas que tematizan los PICS, aspectos de la espiritualidad, así como la relación con la salud integral, resultó ser significativo, como este estudio pretendía presentar a través de la discusión de informes.

Palabras clave: Terapias complementarias; Promoción de la salud; Espiritualidad; Integralidad en salud.

\section{Introdução}

Segundo a agenda de 2030 (United Nations, 2015), em seus Indicadores Brasileiros para os Objetivos de Desenvolvimento Sustentável, preconiza-se dois eixos específicos para tematizar a Saúde e Bem Estar e a Educação de Qualidade, tornando-se relevante a oferta de subsídios para que tais propostas sejam concretizadas.

No que se refere a Salutogênese, ou seja, a busca das razões que levam alguém a estar saudável (Mittelmark et al., 2017), observamos o protagonismo das Práticas Integrativas e Complementares em Saúde (PICS), que privilegiam “à prevenção de doenças e à recuperação da saúde, com ênfase na escuta acolhedora, no desenvolvimento do vínculo terapêutico e na integração do ser humano com o meio ambiente e a sociedade" (Política Nacional de Práticas Integrativas e Complementares no SUS, 2006). Segundo as recomendações da "Declaração de Alma-Ata", a atenção primária à saúde (APS) tem passado por um período de transformações globais, as quais norteiam a implantação das Medicinas Tradicionais, Complementares e Integrativas, que (no Brasil) tem sido fomentada e consolidada por meio das PICS (Barbosa et al., 2020).

De acordo com a Política Estadual de Práticas Integrativas e Complementares (PEPIC) no âmbito da Rede de Serviços do Sistema Único de Saúde (SUS) no Estado do Rio Grande do Norte, acompanhamos avanços significativos a despeito do cenário sanitário e das PICS. “As Práticas Integrativas e Complementares em Saúde devem ser incorporadas na Atenção Básica, Média e Alta Complexidades, inclusive nos Programas Nacionais de Saúde na Escola, Saúde Prisional, Saúde Mental, prioritariamente com ênfase na Atenção Básica a nas Estratégias de Atenção à Saúde da Família” (Lei 10.933, 2021).

Em 1999, a Organização Mundial da Saúde (OMS) passou a descrever qualidade de vida como multidimensional, nas dimensões física, psíquica, social e espiritual (World Health Organization, 1999) (Forti; Serbena; Scaduto, 2020). Compreendendo como definição da espiritualidade uma "conexão com o sagrado, o transcendente e que está intimamente ligada ao sobrenatural, ao místico e a religião organizada" (Isaac; Hay; Lubetkin, 2016), trata-se de uma dimensão intrínseca do ser. Por isso, faz-se pertinente, incluir discussões e olhares para este universo. De acordo com o Manual de Psicologia, Religião e Espiritualidade da APA, é imprescindível explorar os vértices sobre crenças e do sagrado, de forma mais ampla ao longo das formações em saúde (Allicock et al., 2013).

O presente estudo objetivou apresentar relatos de profissionais de saúde, após cursarem um componente sobre as PICS, ofertado pelo Programa de Pós-Graduação em Saúde e Sociedade (PPGSS) da Universidade do Estado do Rio Grande 
do Norte (UERN). Além disso, este estudo buscou unificar temas sobre o ensino, PICS, saúde e espiritualidade mediante os aspectos vivenciais narrados.

\section{Metodologia}

Trata-se de um estudo descritivo, de relato de experiências, desenvolvido por alunos de pós-graduação em saúde que cumpriram com o componente curricular eletivo "Práticas Integrativas e espiritualidade em saúde: Vivências, reflexões e ciência”, do PPGSS. Este componente é ofertado com regularidade anual e contou com pós-graduandos desta instituição e da Universidade Estadual de Campinas, Universidade Federal Rural do Semi-Árido e Universidade Potiguar.

No semestre 2020.2, matricularam-se 33 discentes graduados em diversos cursos da área da saúde. O conteúdo foi estruturado com temáticas sobre as PICS e espiritualidade em diversos âmbitos, abordadas em 20 encontros (60 horas) de modo remoto.

Os encontros foram desenvolvidos a partir de recursos como discussão e debates sobre estudos científicos, seminários, aulas invertidas e expositivas. A aplicação dessas metodologias de ensino buscou estimular reflexões e discussões críticas acerca dos temas.

Esse estudo buscou avaliar relatos vivenciais dos acadêmicos que concluíram a disciplina. Para tanto, os acadêmicos basearam-se em questões norteadoras ${ }^{1}$, definidas pelos autores.

Para manter preservadas as identidades dos participantes, utilizou-se codinomes sobre as temáticas abordadas pelas PICS.

O relato de experiência pode fomentar a realização de iniciativas semelhantes em outras instituições, com adequações à proposta de ensino e inserções de novos conteúdos (Cunha; Scorsolini-Comin, 2019). Esse tipo de estudo permite uma análise crítica e subjetiva dos indivíduos que vivenciaram tal experimentação (Pereira et al., 2018).

\section{Resultados e Discussão}

Após leitura interpretativa dos relatos individuais, emergiram núcleos de sentido que consolidaram categorias de análise independentes. As categorias são: (1) a importância das PICS e da espiritualidade na formação acadêmica, (2) a interdisciplinaridade e aplicações em diferentes contextos e (3) os benefícios inerentes à prática das PICS e da espiritualidade.

\section{PICS e Espiritualidade na formação base}

As PICS constituem um grupo de técnicas embasadas em saberes milenares, cujo principal foco é a contribuição para o autoconhecimento e a qualidade de vida. Essas práticas possibilitam a relação entre o ser e o ambiente no qual está inserido, o conhecimento das interações entre os campos físico, psíquico, energético e espiritual, bem como a promoção da saúde, a partir de um olhar holístico e empoderado para o mundo (Lima; Silva; Tesser, 2014).

A presente categoria tematiza como o contato com as PICS durante a formação base de diferentes cursos em saúde.

Sou formado em Medicina (...). Meu currículo foi baseado nas diretrizes curriculares para o curso de medicina de 2001, com nenhum componente curricular relacionado às PICS e Espiritualidade (...) (Espiritualidade, 2021).

Formado no curso de nutrição e pós graduado em saúde da família, não tive a oportunidade de conhecer as práticas integrativas em nenhum dos cursos (...) (Humanescência, 2021).

\footnotetext{
1 Relatos completos disponíveis em: <https://docs.google.com/document/d/12ahbkJY6_SUo pC3BcEOjyrkX 5MoWg M3bcNz Ijnvlog/edit?usp=sharing)>.
} 
O aprofundamento e o conhecimento sobre a temática das PICS só aconteceu na disciplina de PICS e espiritualidade em saúde, ofertada pelo programa de mestrado em saúde e sociedade da UERN (...) (Resiliência, 2021).

Os relatos despertam a necessidade de ampliação sobre o conhecimento das PICS e espiritualidade na formação acadêmica. Analisando os relatos foi possível inferir que o conhecimento sobre as ferramentas de saúde integral, pode otimizar a manutenção da saúde e, em estados de doença, garantir eficácia terapêutica. Estes saberes podem contribuir para a saúde mental e corporal de acadêmicos.

$\mathrm{Na}$ ausência de estímulos acadêmicos, o aprofundamento no estudo das PICS na formação inicial e a inserção das práticas no campo de trabalho, na maioria dos casos, parte da iniciativa do próprio profissional (Tesser; Souza; Nascimento, 2018). A maioria dos participantes relatou não ter tido contato prévio, com as PICS e os benefícios da espiritualidade durante a formação; inclusive, a busca por conhecer a temática partiu de desejos pessoais.

Contudo, é possível compreender um percurso onde mesclam-se os desafios e conquistas já alcançadas acerca da inserção das PICS no contexto formativo em saúde. E ainda que haja lacunas sobre PICS na graduação, já existem iniciativas assertivas e inclusivas que disseminam tais conhecimentos, como o componente ofertado.

\section{A interdisciplinaridade e a aplicabilidade das PICS e da Espiritualidade}

A interdisciplinaridade pode ser validada mediante atuação multiprofissional e uso de diferentes recursos terapêuticos inerentes às PICS, proporcionando a prevenção, promoção, manutenção e recuperação da saúde, bem como o autocuidado de maneira integrada e humanescente (Nelson, 2019).

Um dos pontos mais marcantes no curso da disciplina foi ver a interação entre diferentes campos do saber, ou seja, a interdisciplinaridade, e os olhares tão diversos que se somaram, frente aos diferentes temas que foram abordados durante o curso (...)" (Confiança, 2021).

Com o objetivo de estimular a interdisciplinaridade e a troca de experiências, o componente curricular permitiu o contato entre profissionais de diferentes áreas de atuação em saúde e regiões do Brasil.

Em relação à aplicabilidade das PICS e da espiritualidade, os relatos evocaram os benefícios do uso desses recursos, tanto os pacientes e comunidade em geral quanto os próprios sujeitos que conhecem suas potencialidades e as aplicam em seu cotidiano.

A disciplina de PICS e espiritualidade em saúde me permitiu conhecer mais sobre as práticas, tendo como desafio encontrado, no transcurso do componente curricular, entender a relação da espiritualidade com a ciência, sendo estas tão distintas (Equilíbrio, 2021).

A afinidade com a temática me impulsionou, enquanto profissional médico e ser humano, crente nos diversos saberes, a buscar um olhar integral e espiritualizado das relações humanas e da vida (...).” (Espiritualidade, 2021)

Fui marcado pela abordagem sobre a meditação, visto que foi uma prática que me permitiu resgatar propósitos, aliviar sofrimentos mentais (como ansiedade e estresse do cotidiano), melhorar a minha capacidade em manejar tarefas e, especialmente, fortalecer a minha espiritualidade (Resiliência, 2021).

Particularmente, as narrativas trazem uma perspectiva de "obscuridade" sobre a relação espiritualidade e ciência. Neste sentido, os profissionais mostraram-se instigados a compreender e buscar por conhecimentos científicos que expandem esta relação causal entre o fortalecimento da espiritualidade e a melhoria da saúde.

Estudos mostram que a espiritualidade é um fator intrínseco ao processo cultural dos seres humanos e que, portanto, deve ser abordada durante o transcurso diagnóstico e terapêutico em contextos de aplicação clínica (Cunha \& ScorsoliniComin, 2019; Leite \& Seminotti, 2013). Isso porque a espiritualidade influencia os hábitos de vida, bem-estar, comportamentos e, ainda, pode modular a qualidade de vida e como o indivíduo enfrentará situações de saúde e adoecimento 
(Cunha \& Scorsolini-Comin, 2019). Nesse sentido, a inserção de componentes curriculares oportuniza, aos acadêmicos, reconhecer as dimensões que ultrapassam o corpo orgânico e os fazem adquirir habilidades no reconhecimento de peculiaridades dos sujeitos envoltos no processo de manutenção e/ou recuperação da saúde (Silva et al., 2020).

Ressalta-se, ainda, que o desenvolvimento espiritual dos acadêmicos de pós-graduação, à luz de seus relatos, faz-se importante para o crescimento pessoal e profissional, implicando positivamente na abordagem ao paciente. De acordo com Aguiar (2013), essa extensão do conhecimento para planos de ordem espiritual confere, ao profissional da saúde, maior liberdade e equilíbrio emocional, tornando-o capaz de ofertar um tratamento holístico aqueles que sofrem; para Silva e colaboradores (2020), este cenário permite um cuidado humanizado, crucial em instituições de ensino e serviços de saúde.

Por fim, sabe-se que o profissional tem a capacidade de se posicionar sobre o seu domínio espiritual, de modo a melhor entender as suas peculiaridades internas e com o sagrado (Cunha \& Scorsolini-Comin, 2019). Contudo, em plena atuação, ele deve manter os seus posicionamentos, crenças e opiniões aliados a uma postura ética, respeitosa e acolhedora, sobretudo diante de embates sobre questões de ordem espiritual e/ou religiosa evocados dos pacientes, familiares e/ou profissionais.

\section{Os benefícios das PICS na vida pessoal, acadêmica e profissional}

Os benefícios proporcionados pelas PICS repercutem na esfera profissional, no setor pessoal, contribuindo para o desenvolvimento de uma melhor relação consigo e com o mundo, e no ambiente acadêmico.

Os relatos que fundamentaram esta categoria permitem inferir que, no âmbito profissional, a ampliação do conhecimento sobre as PICS expandiu o campo de possibilidades terapêuticas, possibilitando a inserção de novas ferramentas e técnicas que fortalecem o vínculo com o paciente e contribui para a melhoria na qualidade de vida, adesão ao tratamento e a obtenção de melhores resultados, como versa o seguinte relato:

(...) profissionalmente é que posso referir o maior impacto, pois hoje tenho um arsenal de possibilidades terapêuticas mais amplo para tratar meus pacientes oncológicos e discutir com os colegas da clínica. E considerando que este sempre foi meu objetivo: melhorar a qualidade do meu atendimento farmacêutico, é este meu grande prêmio nesta disciplina (...) (Ressignificação, 2021).

Os participantes do estudo descreveram os benefícios alcançados na esfera pessoal, podendo ser entendidos a partir da perspectiva do seguinte relato:

A meditação, a reflexologia e a aplicação de técnicas de respiração, são instrumentos que marcaram e passaram a integrar o meu dia a dia, possibilitando sempre voltar ao eixo e ao equilíbrio diante da rotina. Os benefícios gerados na concentração, foco e no autoconhecimento, permitindo entender melhor as emoções e comportamentos (Humanescência, 2021).

Em geral, foi relatada a melhoria no autoconhecimento e autocuidado, o que é essencial, especialmente considerando os quadros de desordens psicológicas que foram exacerbados entre os profissionais de saúde durante a pandemia de COVID-19 (Dantas, 2021) (Moreira; Souza; Nobrega, 2020).

No âmbito profissional e acadêmico, as narrativas corroboram com o estudo de Silva et al. (2021). Esses autores observaram que os profissionais de saúde geralmente financiam a própria formação nas Práticas Integrativas e Complementares; financiamento é motivado pelo reconhecimento da importância do manejo das PICs para manutenção da própria saúde e da saúde de seus pacientes (Silva et al., 2021). De modo geral, esse cenário expõe a falta de iniciativas de educação em saúde sobre as PICs, para a qualificação de profissionais; o que também foi retratado nas narrativas apresentadas por este estudo, uma vez que a maioria dos acadêmicos teve o seu primeiro contato no componente curricular ofertado pelo Programa de Pós-graduação em Saúde e Sociedade (UERN). 
Por fim, reconhecemos que o conhecimento das PICS impactou positivamente a saúde global destes profissionais, bem como permitiu maior crescimento acadêmico e melhor qualidade dos atendimentos em serviços de saúde.

\section{Considerações Finais}

De acordo com as vivências e anseios dos profissionais, percebe-se que a inserção das PICS e espiritualidade, na graduação, apresenta-se como falta, mesmo diante de iniciativas em programas de pós-graduação. Este cenário evoca mudanças nas matrizes curriculares dos cursos de graduação em saúde.

As experiências adquiridas na disciplina propiciaram uma visão integral sobre as necessidades humanas, o que instigou os profissionais a pensarem criticamente sobre construções afirmativas na integração entre o usuário, o profissional e o serviço de saúde. As narrativas mostraram que a aplicação das PICS e da espiritualidade impactou os profissionais positivamente em vários âmbitos de suas vidas.

Com base no que foi apresentado ao longo do presente estudo, aponta-se como necessária, a ampliação de novos estudos que abordem os impactos dos conhecimentos das PICS, na formação pessoal e laboral, entre diferentes profissionais de saúde, de modo a conceber novos saberes acerca da concepção de saúde, para além do processo de doença, viabilizando o bem estar e qualidade de vida dos sujeitos atendidos pelo campo assistencial.

\section{Agradecimentos}

Agradecemos ao Programa de Pós-Graduação em Saúde e Sociedade (PPGSS) da Universidade do Estado do Rio Grande do Norte (UERN) pela possibilidade de troca de conhecimentos sobre as PICS, entre diferentes profissionais da saúde. Agradecemos à Fundação de Amparo à Pesquisa do Estado de São Paulo (FAPESP) e à Coordenação de Aperfeiçoamento de Pessoal de Nível Superior (CAPES), pelo fomento concedido à dois, dos autores do presente artigo.

\section{Referências}

Aguiar, L. S. F. (2013). Uma proposta para a espiritualidade na formação médica. In: Salgado, MI., Freire, G. Saúde e Espiritualidade - Uma nova visão da Medicina. São Paulo: Editora Inede. p. 31-42.

Allicock M., Resnicow K., Hooten, E. G, \& Campbell, M. K. (2013). Fé e comportamento de saúde: O papel da igreja afro-americana na promoção da saúde e prevenção de doenças. In: Pargament Kenneth I, Mahoney Annette, Shafranske Edward P., editores. Manual da APA de psicologia, religião e espiritualidade (Vol 2): Uma psicologia aplicada da religião e espiritualidade. Manuais da APA em psicologia. Washington, DC, EUA: American Psychological Association, p. $439-459$

Barbosa, F. E. S., Guimarães, M. B. L., Santos, C. R., Bezerra, A. F. B., Tesser, C. D., \& Sousa, I. M. C. (2020). Oferta de Práticas Integrativas e Complementares em Saúde na Estratégia Saúde da Família no Brasil. Cadernos de Saúde Pública, 36 (1), 1-13.

Cunha, V. F., \& Scorsolini-Comin, F. (2019). A religiosidade/espiritualidade (R/E) como componente curricular na graduação em Psicologia: relato de experiência. Psicologia Revista, 28 (1), 193-214.

Cunha, V. F., \& Scorsolini-Comin, F. (2019). A dimensão religiosidade/espiritualidade na Prática Clínica: revisão Integrativa da literatura científica. Psicologia: Teoria e Pesquisa, 35, 1-12.

Dantas, E. S. O. (2021). Saúde mental dos profissionais de saúde no Brasil no contexto da pandemia por COVID-19. Interface (Botucatu)., 25 (supl.1).

Forti, S., Serbena, C. A., \& Scaduto, A. A. (2020). Mensuração da espiritualidade/religiosidade em saúde no Brasil: uma revisão sistemática. Ciênc. saúde coletiva, 25 (4), 1463-1474.

Isaac, K. S., Hay, J. L., \& Lubetkin, E. I. (2016). Incorporating Spirituality in Primary Care. J Relig Health, 55 (3), $1065-1077$.

Lei n ${ }^{\circ}$ 10.933. (2021). Disponível em: <http://diariooficial.rn.gov.br/dei/dorn3/docview.aspx?id_jor=00000001\&data=20210618\&id_doc=727178>.

Leite, I. S., \& Seminotti, E. P. (2013). A influência da espiritualidade na prática clínica em saúde mental: uma revisão sistemática. Rev. bras. ciênc. saúde, 17 (2), 189-196.

Lima, K. M. S. V., Silva, K. L., \& Tesser, C. D. (2014). Práticas integrativas e complementares e relação com promoção da saúde: Experiência de um serviço municipal de saúde. Interface Commun Heal Educ, 18 (49), 261-72. 
Research, Society and Development, v. 10, n. 7, e59510717114, 2021

(CC BY 4.0) | ISSN 2525-3409 | DOI: http://dx.doi.org/10.33448/rsd-v10i7.17114

Mittelmark, M. B., Sagy, S., Eriksson, M., Bauer, G. F., Pelikan, J. M., Lindström, B., \& Espnes, G. A. (Eds.). (2017). The Handbook of Salutogenesis. Springer.

Moreira, W. C., Souza, A. R., \& Nobrega, M. P. S. S. (2020). Mental illness in the general population and health professionals during COVI-19: a scoring review. Texto \& contexto enferm, 29, 1-17.

Nelson, I. C. A. S. R. (2019). As práticas integrativas e os cuidados humanescentes em saúde. EDUERN.

Pereira, A. S., Shitsuka, D. M., Pereira, F. J., \& Scitsuka, R. (2018). Metodologia da pesquisa científica. UFSM.

Política Nacional de Práticas Integrativas e Complementares no SUS. (2006). 〈https://aps.saude.gov.br/ape/pics〉.

Silva, L. L., et al. (2020). Espiritualidade, saúde e cuidado humanizado em ambiente de ensino: relato de experiência. Disciplinarum Scientia| Saúde, 21 (2), $11-18$.

Silva, P. H. B., et al. (2021). Formação profissional em Práticas Integrativas e Complementares: o sentido atribuído por trabalhadores da Atenção Primária à Saúde. Ciência \& Saúde Coletiva, 26, 399-408.

Tesser, C. D., Sousa, I. M. C. de, \& Nascimento, M. C do. (2018). Práticas Integrativas e Complementares na Atenção Primária à Saúde brasileira. Saúde em Debate, 42 (spe1), 174-88.

United Nations. (2015). Transforming our world: the 2030 agenda for sustainable development. ONU. Retrieved from https://sustainabledevelopment.un.org/post2015/transformingourworld/publication.

World Health Organization (WHO). (1999). Fifty-second World Health Assembly, Geneva, 17-25 May 1999: verbatim records of plenary meetings and list of participants. https://apps.who.int/iris/handle/10665/260180. 